\title{
La Cohesión Regional en los Procesos de Integración en América Latina y el Caribe
}

\author{
Ignacio Bartesaghi ${ }^{1}$, María Eugenia Pereira ${ }^{{ }^{*}}$
}

Resumen: El artículo explora la situación actual de tres procesos de integración latinoamericana: el ALBA, la Alianza del Pacífico y el MERCOSUR, a través de un relevamiento cualitativo de los logros obtenidos en relación a los objetivos fijados inicialmente por cada uno de ellos. El trabajo se enmarca en analizar el liderazgo y la innovación de los mencionados procesos, ya que todas las relaciones entre los Estados miembros de los distintos esquemas de integración analizados, como la de estos entre sí, necesitarán de un fuerte liderazgo e innovación, para enfrentar de forma satisfactoria los importantes desafíos futuros que impone el nuevo contexto internacional para alcanzar un mundo sustentable.

\begin{abstract}
Through a quantitative survey of the current status of all the integration processes in the region, the study explore the founding objectives and aims finally achieved by three integration models in the region: ALBA, Pacific Alliance and MERCOSUR. This article focuses on leadership and innovation for a sustainable world because the relation between the Members of the different integration processes will need a strong leadership and innovation policy to face successfully the important future challenges posed by the new international environment to achieve a sustainable world.
\end{abstract}

Keywords: Economic Integration; Cohesion, Institutions; MERCOSUR; ALBA; Pacific Alliance

$21^{\text {st }}$ International Association of Jesuit Business Schools (IAJBS)

$18^{\text {th }}$ Colleagues in Jesuit Business Education (CJBE)

\section{Introducción}

Desde la segunda mitad del siglo XX, América Latina ha sido una de las regiones más dinámicas en la implementación de procesos de integración, muchos de los cuales siguieron el modelo europeo a partir de la conformación de la Comunidad Económica Europea en el año 1957.

Si bien la mayor parte de los procesos marcaron objetivos económicos y comerciales, debido a la profundidad institucional que adquirieron los mismos, pronto comenzaron a incorporar otros que van más allá de dichos ámbitos y abarcan otras áreas que podrían favorecer, de tener éxito, la cohesión de los miembros frente al proceso de integración.

En lo últimos años han proliferado un importante número de iniciativas de integración en América Latina, algunas de ellas con perfiles económicos, otras con perfiles sociales o políticos. Incluso ciertos ámbitos fueron presentados como contrapuestos o sustitutivos, en un contexto marcado por el no cumplimiento de algunos de los objetivos originarios definidos por los procesos de integración.

A través de un relevamiento cualitativo de las principales características de todos los procesos de integración de la región, y llevando adelante un estudio comparativo entre tres de ellos, Mercosur, ALBA y a la Alianza del Pacífico, se pretende analizar las principales características de cada proceso y los avances en cuanto al cumplimiento de sus objetivos fundacionales, aspecto clave para alcanzar la cohesión de los miembros en cada proceso, así como el respeto a las instituciones regionales.
América Latina atraviesa ciertas dificultades en todos los instrumentos de integración vigentes, incluso con algunos posibles desmembramientos institucionales que no favorecen la estabilidad necesaria para que la región siga adelante con aquellas reformas que permitirán un desarrollo sustentable. El estudio comparativo de algunos de los procesos de integración de mayor impacto regional en los últimos años, pretende identificar si las innovaciones seguidas por algunos ámbitos integracionistas, lograrán superar las diferencias nacionales para consolidar una visión regionalista que haga posible una verdadera integración entre los países de América Latina y el Caribe.

\section{Los cimientos de la integración regional}

El origen de los cimientos de la integración comercial entre Estados Nacionales se remonta a algunos siglos atrás, con el dominio del libre comercio impulsado por Inglaterra y por los impactos de la Revolución Industrial, que forzaron la búsqueda de nuevos mercados. De cualquier forma, no fue hasta bien entrado el siglo XX, particularmente una vez se alcanzó una relativa estabilidad institucional a nivel internacional, que el fenómeno de la integración logró consolidarse como un camino sin retorno.

Es en este marco que terminan de emerger los organismos internacionales, como las Naciones Unidas, el Banco Internacional de Reconstrucción y Fomento, conocido hoy como Banco Mundial, el Fondo Monetario Internacional y el intento por conformar la Organización Internacional del Comercio, que derivó en un Acuerdo General de Aranceles y Comercio (GATT en sus siglas en inglés), hoy transformado en la Organización Mundial del Comercio (OMC), institución

(1) Departamento de Negocios Internacionales e Integración, Facultad de Ciencias Empresariales, Universidad Católica del Uruguay.

*Corresponding autor: mariaeugenia.pereira@ucu.edu.uy 
encargada de reglar las disciplinas comerciales a nivel internacional y la que de acuerdo a la misma organización ha cumplido un rol en la estabilidad política internacional (OMC, 2011).

Las mencionadas organizaciones internacionales fueron transformando el concepto que, por su origen y evolución histórica, hizo que el Derecho Internacional aceptara solo a los Estados como únicos sujetos del mencionado derecho (Diez de Velasco, 2002). Además, la consolidación de esas iniciativas de fuerte raíz política, permitieron la instalación definitiva de los procesos de integración, los cuales hicieron posible un acercamiento definitivo entre aquellos Estados enfrentados.

La Comunidad Económica del Carbón y del Acero (CECA) constituida por el Tratado de París de 1951, inició el acercamiento definitivo entre Francia y Alemania, que a través de un fuerte liderazgo político de grandes ideólogos integracionistas, constituyeron el original instrumento que administraba de forma conjunta dos recursos claves, dado la importancia económica, el número de empleos directamente relacionados con la industria y su relación con la producción de armas. Este fue sin lugar a dudas el inicio de un camino sin retorno hacia la estabilidad de Europa, resguardada por Estados Unidos y los innegables efectos del Plan Marshall. Pronto se cerró el acuerdo de mayor referencia a nivel internacional hasta el presente, la Comunidad Económica Europea (CEE), aprobada por el Tratado de Roma de 1957, hoy Unión Europea (Bartesaghi, 2012).

Como se señalará en los capítulos siguientes, la CEE favoreció la implementación de un modelo de integración que pronto fue seguido por otras regiones que atravesaban también diferentes problemáticas sociales, económicas y políticas, caso de América Latina, África o el Sudeste asiático. Si bien es cierto que los Estados tenían la convicción de que los instrumentos de integración económica eran vehículos adecuados para generar riqueza y crecimiento, como por ejemplo el esperado efecto en las economías de escala y aumento de la eficiencia por el aumento del mercado, también fue evidente la importancia de los procesos de integración en términos políticos y sociales, en particular en aquellos casos donde dichos instrumentos jugaron un papel trascendental en la estabilidad política, donde el instrumento de integración visualizado como un mecanismo supranacional, permitió superar las diferencias internas entre los Estados vecinos. Por lo tanto, los procesos de integración jugaron y siguen jugando un rol fundamental en la estabilidad internacional y en la cohesión regional, único camino posible para el desarrollo sustentable de las economías.

Como se verá en apartado siguiente, los modelos de integración desplegados por los Estados latinoamericanos son variados en sus intereses y perfiles, algunos centrados en la integración económica, otros actuando como foros políticos, o atendiendo a temas de desarrollo, pero también existen los que potencian la cooperación. Más allá de estas distinciones entre los diferentes procesos, la multiplicación de las iniciativas llevó a que muchos de los objetivos de éstas se super- pongan, lo que afectó en definitiva el cumplimiento de las metas originarias de las mismas, generalizando en algunos ámbitos la posición de que la integración no cumple por definición con sus objetivos. Generalizar este concepto, se trata no solo de un error conceptual, sino también de un riesgo para la estabilidad regional, la que continúa dependiendo de la existencia de estas iniciativas, más allá de sus falencias. En un desafío y una obligación de los Estados, evitar que estas percepciones se consoliden en América Latina y el Caribe, lo que solo es posible con acciones concretas en pos de cumplir con lo que se acuerda.

\section{Fenómeno de la integración regional en América Latina y el Caribe ${ }^{1}$}

Al principio de la segunda mitad del siglo XX, los países latinoamericanos encontraron buenos motivos para iniciar las negociaciones comerciales regionales, alentados por las políticas de la CEPAL, quien argumentaba que a través de este medio, era posible lograr el tan anhelado desarrollo industrial para América Latina (Quitral Rojas, 2009).

El primer proceso de integración nacido en este período fue la Asociación Latinoamericana de Libre Comercio (ALALC), nacida bajo el Tratado de Montevideo en 1960. En el mismo, se propuso alcanzar un área de libre comercio en un plazo de doce años, la que nunca llego a concretarse, marcando así, el final de esta organización y el nacimiento de la Asociación Latinoamericana de Integración (ALADI) con el Tratado de Montevideo de 1980, proceso con objetivos más flexibles que mantiene su vigencia hasta el presente.

A principio de la década del 60 nace también el Mercado Común Centroamericano (MCCA) al firmarse el Tratado General de Integración Económica (conocido también como el Tratado de Managua) en el que Guatemala, El Salvador, Honduras, Nicaragua y Costa Rica se comprometieron a conformar un mercado común a través de la libre circulación de los factores productivos. Si bien en un inicio este proceso fue señalado como un ejemplo a seguir por la región, con el correr de los años los problemas internos entre sus miembros (conflicto entre El Salvador y Honduras, en 1969), así como problemas climáticos (terremotos en Nicaragua y Guatemala, el huracán Fifi que afectó a Honduras), así como falta de voluntad política de algunos de sus miembros a la hora de aplicar el arancel externo común, hicieron que MCCA entrase en una fase de estancamiento. Con el protocolo de Guatemala, en el año 1993, los presidentes centroamericanos realizan una fuerte apuesta a la integración regional, marcando así el relanzamiento del Mercado Común Centroamericano (López, 2012).

Se debe mencionar también la formación del Sistema de Integración Centroamericana (SICA) en el 1991 con el protocolo de Tegucigalpa, firmado por los cinco miembros del MCCA más Panamá, quien le dio un marco institucional a la integración centroamericana. Posteriormente, Belice (2000) y República Dominicana (2013) se adhirieron a este proceso de integración.

1 Se consideraron todos los procesos de integración de base económica y comercial, descartando los que se encuentran en el ámbito político y operan como foros políticos. Asimismo se descartó la incorporación de instituciones sectoriales. 
Cabe precisar que se trata de un acuerdo profundo en términos de sus objetivos e instituciones, ya que siguió el modelo europeo de integración. En efecto, el bloque cuenta con un sistema institucional compuesto por una Secretaría con amplias funciones, con un Parlamento Centroamericano y por un Tribunal de Justicia Centroamericano, entre otros órganos. Además, ha logrado negociar de forma conjunta acuerdos comerciales y de asociación con Estados Unidos y la Unión Europea. Si bien con imperfecciones, se trata de uno de los procesos de mayor éxito en el cumplimiento de sus objetivos, especialmente desde su relanzamiento en la década del noventa.

Pocos años después, ya finalizada la década del sesenta, cinco países sudamericanos deciden conformar el Pacto Andino, hoy conocido como Comunidad Andina (CA). El 26 de mayo de 1969, Bolivia, Colombia, Chile, Ecuador y Perú firman el Acuerdo de Cartagena que da origen a este proceso de integración, al que posteriormente, en 1973, se le une Venezuela. Chile abandonó el acuerdo en 1976.

Si bien la CA ha logrado implementar un mercado ampliado sin el pago de aranceles para un $100 \%$ de universo arancelario, en la actualidad y desde la salida de algunos de sus miembros, el caso ya comentado de Chile y el más reciente de Venezuela (concretado en 2006), han desafiado la institucionalidad y continuidad del proceso de integración. Así como ocurrió con el MCCA, se trata de un proceso de integración que apostó a un nivel de integración profundo, replicando muchos de los órganos existentes en la Unión Europea.

La institucionalidad del proceso se estructuró en lo que se conoce como el Sistema Andino de Integración, el que permitió a la CA desarrollar la supranacionalidad de sus órganos, aspecto que se diferencia de otros procesos de integración de la región como por el ejemplo el MERCOSUR. Asimismo, logró la aprobación de normas regionales en áreas que van más allá del plano comercial, como el caso de las relacionadas con la propiedad intelectual, asuntos laborales o medioambientales entre otros. Además, logró la implementación y eficiente funcionamiento de un sistema de solución de controversias contando con un Tribunal de Justicia. A su vez, contó de forma adelantada con un Parlamento y con una Secretaría con funciones que superan ampliamente las tareas administrativas.

Sin embargo, más allá de los logros mencionados, actualmente la CA enfrenta un debilitamiento institucional producido por las diferencias entre los miembros, las que son de índole político, pero no solo, lo que incluso provocó el alejamiento de algunos miembros y la constitución por parte de dos de sus socios fundadores (Colombia y Perú) de una iniciativa regional en paralelo, que llega a duplicar parte de los objetivos ya consagrados en la CA. Los dos mencionados Estados justifican este accionar en que el cambio en las relaciones comerciales mundiales de los últimos años y el resurgimiento de las potencias asiáticas, lleva a la necesidad de actuar de forma acelerada para anticiparse a las nuevas dinámicas, lo que no fue posible en el marco de la
CA. Esta nueva realidad llevó al proceso andino a iniciar una reforma institucional que afectará su organigrama actual, en particular la importancia de la Secretaría y el Parlamento.

La región del Caribe no se mantuvo al margen de la ola de la integración económica iniciada a partir de la década del sesenta. En efecto, ya desde 1965 se firmó la Asociación de Libre Comercio del Caribe, conocido como CARIFTA, proyecto que buscó fomentar la integración de los países caribeños tras el fracaso de la Federación de las Indias Occidentales. El CARIFTA buscaba ser una zona de libre comercio entre Trinidad y Tobago, Antigua y Barbuda, Barbados y Guyana. En 1973, tras el Tratado de Chaguaramas, el CARIFTA se convierte en el Mercado Común del Caribe (CARICOM por sus siglas en ingles) con el fin de avanzar en la integración económica comercial de los países que integraban el bloque.

Actualmente este proceso cuenta con 15 miembros, siendo Haití el último país en incorporarse. Entre sus logros más importantes, se puede destacar el Acuerdo de Asociación Económica entre la Unión Europea y el CARIFORUM (espacio integrado por los quince países miembros del CARICOM más República Dominicana) firmado en el 2008, en el que los países caribeños logran dar un paso más de acercamiento con el principal bloque de integración económica, consolidando las preferencias que esta región gozaba con la Unión Europea por la existencia de regímenes excepcionales. Así como ocurre con los otros procesos de integración ya comentados, el CARICOM no está ajeno a las críticas por los escasos resultados obtenidos, no solo en materia comercial, sino en materia de cooperación. A esta situación se le debe sumar la incapacidad de conformar lo pactado de transformar el CARICOM en un mercado único para el 2008 (Romero, 2013).

Este hecho demuestra que el proceso de integración caribeño no escapa al estancamiento que sufren otros bloques de la región, que en ningún caso han podido completar plenamente el objetivo de implementar un mercado común, más allá de que también el bloque caribeño conformó una estructura institucional profunda, principalmente destacable en su sistema de solución de controversias liderado por la Corte de Justicia del Caribe, la Secretaría o la Asamblea de Parlamentarios.

Con un nuevo tratado marco, tras la firma del Tratado de Montevideo de 1980 y la formación de la ALADI, nuevos instrumentos de integración económica fueron puestos al alcance de los países. Esta institución busca formar un mercado común entre los trece países miembros, sin determinar una fecha específica para alcanzarlo. ${ }^{2}$

La ALADI es un proceso de integración que incorporó dos conceptos clave para su desarrollo, el trato especial y diferenciado para los países considerados de menor desarrollo relativo (Bolivia, Ecuador y Paraguay) y la flexibilidad en los instrumentos de integración, conocidos como los acuerdos de alcance regional y parcial, siendo estos últimos

2 Esta definición podría ser considerado una innovación en cuanto a los instrumentos, ya que por el contexto en que se aprobó la ALADI y teniendo en cuenta el antecedente de la ALALC, se intentó evitar la aprobación de una meta concreta que sea nuevamente incumplida, como el caso de la conformación de una zona de libre comercio prevista en el Tratado de Montevideo de 1960. 
los de mayor importancia, ya que permitieron la concreción de un importante número de relaciones subregionales y bilaterales que a la postre llevaron a que gran parte del comercio regional se encuentre liberalizado (cerca del 70\%).

Por otra parte, la ALADI también enfrenta algunos desafíos, en particular en lo que tiene que ver con la aprobación de mecanismos comunes que permitan la armonización mandatada por la Resolución 59 en normas y disciplinas comerciales, en apoyo a los PMDER y en las denominadas disciplinas complementarias. Cabe precisar que el bloque no ha logrado aprobar normas comunes en defensa comercial, solución de controversias, servicios o inversiones.

En la década del noventa se consolida un nuevo paradigma en la integración económica, el que estuvo impulsado por los organismos internacionales (Consenso de Washington) y por el cambio de estrategia de Estados Unidos, el que relegó el ámbito multilateral (Ronda Uruguay del GATT) frente al bilateral y regional. En consecuencia, la primera potencia mundial suscribió un acuerdo bilateral con Canadá, el que luego incorporó a México. Paralelamente impulsó la firma del Foro de Cooperación Económica de Asia Pacífico, conocido como APEC por sus siglas en inglés y lanzó el Área de Libre Comercio de las Américas (ALCA), que buscaba la integración comercial entre todo el continente americano con excepción de Cuba.

El nuevo paradigma de integración tuvo sus efectos en la región, que a partir del regionalismo abierto reformó parte de los procesos de integración creados en décadas anteriores y a su vez constituyó nuevos, caso del MERCOSUR. Además, una de las características de estos procesos tiene que ver con que los mismos incorporarían progresivamente lo que a nivel multilateral se conocía como los nuevos temas del comercio, especialmente de interés para las potencias desarrolladas (Sanahuja, 2007).

En este nuevo contexto surge el MERCOSUR, acuerdo creado por el Tratado de Asunción el 26 de marzo de 1991 firmado por Argentina, Brasil, Paraguay y Uruguay. Este proceso de integración encuentra sus antecedentes en el acercamiento entre Brasil y Argentina, el que permitió una asociación fundamental para dejar de lado cualquier hipótesis de conflicto bilateral. En ese sentido, a través de la firma de la Declaración de Iguazú (1985), el Programa de Integración Argentino- Brasileño, conocido como el PICAB, la Declaración de Buenas Aires y la firma del Acuerdo de Complementación Económica en el marco de la ALADI, las dos potencias de la región iniciaron el proceso de gestación del MERCOSUR, al que de forma tardía se sumaron Uruguay y Paraguay (Bartesaghi, 2011).

En sus 24 años de existencia, el bloque ha atravesado por diferentes circunstancias que marcaron su rumbo. Su tratado originario plantea en su artículo primero la meta de alcanzar un mercado común, lo que implica la libre movilidad de los factores de producción, para lo cual previamente establecería la libre circulación de bienes y servicios y la conformación de un arancel externo común. Más allá del dinamismo inicial mostrado por el MERCOSUR, principalmente en términos de las rebajas arancelarias que redundó en el indiscutible aumento del comercio intrarregional, el bloque siguió la tónica de los otros procesos en el incumplimiento de gran parte de sus objetivos originarios, especialmente desde el año 1999 en adelante (devaluación de Brasil). $\mathrm{Al}$ respecto de las metas propuestas, cabe aclarar que lo llamativo de este proceso de integración tiene que ver con los plazos, ya que se planteó la concreción de un nivel de integración en un período de años muy breve en términos comparados, lo que en definitiva adelantaba desde sus orígenes el resultado que finalmente se alcanzaría (Bartesaghi, 2015).

De todas formas, logró con éxito su profundización institucional con la aprobación del Protocolo de Ouro Preto, por el cual se establece una estructura orgánica y se le otorga personalidad jurídica internacional. Con la aprobación del Protocolo de Brasilia y posteriormente el de Olivos del año 2002, se establece el Sistema de Solución de Controversias, el que presenta distintas características al del proceso andino, centroamericano y caribeño, ya que no cuenta con un Tribunal de Justicia regional, lo que tiene que ver con el grado de integración real alcanzado por los Estados miembros. Además de una Secretaría ${ }^{3}$, el bloque creó un Parlamento, que sustituyó a la Comisión Parlamentaria Conjunta (Bartesaghi, 2011).

La densidad institucional presentada, es un ejemplo de cómo el proceso sigue luego de varias décadas el modelo europeo, vigencia que también se hizo evidente con la reforma de los procesos de integración de la región recién mencionados, donde en plena crisis institucional se decidió profundizar las instituciones en lugar de debilitarlas. De cualquier forma, cerca de llegar al cuarto de siglo de existencia, el MERCOSUR no escapa a la situación de estancamiento que atraviesan otros procesos de integración de estas características en la región, si bien no con intensidad de la CA.

Algunas de las dificultades recientes del bloque tienen que ver con el ingreso de Venezuela al bloque, en momentos en que Paraguay se encontraba suspendido, alegándose que lo "político está por encima de lo jurídico" en una frase que refleja la situación actual del MERCOSUR. Además, no logró alcanzar una unión aduanera perfeccionada, ya que está en funcionamiento pero con un sin número de excepciones, no avanzó en la implementación del mercado común y tampoco ha negociado normas regionales en las disciplinas de mayor importancia para el comercio internacional en la actualidad, caso de las inversiones, servicios, propiedad intelectual, medio ambiente, entre otras.

Otro de los problemas que atraviesa el bloque tiene que ver con su agenda externa, que muestra escaso dinamismo en la suscripción de acuerdos con otros países y regiones. Cabe precisar que este es un aspecto de suma importancia para la cohesión e institucionalidad del

3 La secretaría del MERCOSUR atravesó por diferentes etapas. Inicialmente fue solo administrativa, luego se la dotó de técnicos para que asesoren a los gobiernos en el desarrollo del proceso y elaboren estudios, lo que fue descartado por intereses políticos. En la actualidad se trata de una secretaría con escasas capacidades técnicas, dedicada a los aspectos administrativos y organizativos. Con la creación del cargo del Alto Representante del MERCOSUR, se le otorgó cierta función política. 
bloque, ya que algunos países como Uruguay, en su condición de país pequeño, depositaron su expectativa en que el bloque serviría como plataforma para insertarse en el mundo, lo que a la postre no sucedió. Lo ocurrido con la CA en términos de su desmembramiento institucional y pérdida de alguno de sus miembros, demuestra cómo las diferencias políticas en la estrategia de inserción seguida por los bloques (los que a este nivel de integración se supone al menos teóricamente debe ser conjunta), puede llevar a quiebres en los procesos, si no se actúa con innovación en términos de las flexibilidades exigidas por los contextos, nacionales, regionales e internacionales. En este punto, tanto el CARICOM como el MCCA han tenido mayor éxito, ya que han logrado en relacionarse de forma conjunta con Estados Unidos y la Unión Europea.

\section{¿Nuevos procesos de integración?}

El cambio de siglo trajo consigo fuertes movimientos en el escenario político de la región, que en términos políticos fue definido como una nueva ola de izquierda y en algunos casos con gobiernos de corte populista.

Este nuevo movimiento en la región logró su fortaleza con las acciones contrarias a la gran potencia en diferentes ámbitos, especialmente en las negociaciones para la conformación del ALCA, opción que fue desarticulada silenciosamente por Brasil, pero mediáticamente por Venezuela, que con el Presidente Chávez ya adquiría un perfil de país enfrentado con Estados Unidos en su corrida antiimperialista. Con una Ronda Doha lazada en 2001 en serias dificultades y con la estrategia continental paralizada por algunos países de la región, EEUU debió cambiar su estrategia hacia la firma de acuerdos bilaterales, con aquellos países que en el proceso de la negociación del ALCA habían dado señales políticas de acercamiento con la primera potencia mundial. En ese contexto, suscribió un acuerdo con Centroamérica conocido como CAFTA - RD, el que negoció conjuntamente en lo que fue considerado un éxito para la administración de Bush (h) y logró suscribir acuerdos bilaterales con Chile, Perú y Colombia. En dos oportunidades, si bien siendo procesos con características propias, lo intentó con Uruguay.

La estrategia norteamericana llevó a que en la región se generara cierta división política respecto a EEUU, lo que se vio potenciado por la política exterior seguida por Bush (h) en el marco de la denominada guerra contra el terrorismo. Como consecuencia, especialmente Chávez implementó una estrategia diplomática que tuvo por objetivo alinear a los países en bloques antiimperialistas o socialistas, rememorando la lógica de la Guerra Fría, marco en el cual Venezuela propone en 2004, la creación de la Alianza Bolivariana para los pueblos de nuestra América (conocido por sus siglas ALBA). Esta organización, que es un fiel reflejo de la politización que vivió la integración en América Latina durante los últimos quince años, nace de un acuerdo entre Cuba y Venezuela, al que se les unirá Bolivia en el año 2006. Actualmente está compuesto por once miembros, que además de los tres ya mencionados son también parte del ALBA, Antigua y Barbuda, Dominica, Ecuador, Granada, Nicaragua, San Cristóbal y Nieves, San Vicente y las Granadinas y Santa Lucía, lo que le otorga una repre- sentatividad geográfica amplia, tanto en términos de sus miembros, como en una región de importancia para EEUU.

Entre los objetivos principales se destacan la lucha contra la pobreza y la exclusión social, contemplando las asimetrías existentes en los países que componen el bloque y buscando mecanismos de reducción de las mismas. Este foro político cuenta con un acuerdo que cubre el área económica comercial denominado Tratado de Comercio de los Pueblos. A pesar de esto, la organización no cuenta con personalidad jurídica internacional, por lo que cualquier tratado que quieran firmar los países miembro tiene que ser de forma bilateral (SELA, 2014).

En el año del 2009, surge la Unión Sudamericana de Naciones (UNASUR) un nuevo espacio liderado por Brasil que busca incluir a los países de América del Sur en un foro político bajo la tutela del gigante sudamericano. La UNASUR es la sucesora de la Comunidad Sudamericana de Naciones que tenía en sus orígenes un enfoque más comercial tendiente a la convergencia entre los dos procesos de integración de mayor importancia - al menos hasta la fecha - de Sudamérica, como son la CA y el MERCOSUR.

Actualmente los miembros que componen este bloque son Argentina, Bolivia, Brasil, Chile Colombia, Ecuador, Guayana, Paraguay, Perú, Surinam, Uruguay y Venezuela. Los objetivos de la UNASUR son de carácter político, desatancándose entre los mismos la cooperación en sus diversas áreas, el fortalecimiento del diálogo político, la consolidación de la identidad sudamericana y el desarrollo de mecanismos concretos para la superación de las asimetrías. ${ }^{4}$

Frente a este escenario en términos de bloques con un importante sesgo político y geopolítico, se gestó una estrategia de algunos países de la región, que no compartían la visión de Venezuela respecto a su retórica anti norteamericana, pero tampoco la de Brasil en la región, la que este país visualiza sin la presencia de México que se sintió excluido de la UNASUR.

Ese en este contexto regional que se abre espacio para a Alianza del Pacífico (proyecto lanzando en la Declaración de Lima del año 2009), el que contó con el impulso de Perú y fue acompañado por Chile y Colombia. Si bien es México el último país en acompañar la iniciativa, su incorporación termina de consolidar a la Alianza como un proyecto de impacto regional y global, ya que el país norteamericano que no había sido integrado en la lógica sudamericana que impulsaba Brasil a través de la UNASUR, ingresa nuevamente al escenario sudamericano, estrategia que acompaña con una mayor presencia en la ALADI, otro ámbito integracionista que lo relaciona con las economías sudamericanas.

Entre sus objetivos se destacan la conformación de un especio de libre circulación de bienes, servicios y factores de producción (lo que sería definido por la teoría clásica de la integración como un mercado común), pero sin la conformación previa de una unión aduanera. Desde su constitución, la Alianza del Pacífico se mostró como un proceso moderno de integración en términos de su pragmatismo, flexibilidad e instrumentos. Como se acaba de mencionar, su tratado originario no menciona como meta ningún nivel de integración conocido por

4 Tratado constitutivo de la Unión de Naciones Suramericanas. 
la teoría clásica, tampoco establece metas concretas que lleven a un incumplimiento como el que caracteriza a gran parte de los objetivos planteados por los otros procesos ya reseñados en este capítulo.

Con un claro enfoque económico y no político (característica de la mayor parte de las iniciativas nacidas en el siglo XXI en América Latina), la Alianza del Pacífico se ha posicionado como un nuevo modelo de inserción externa en la región, donde no solo ya cuenta con acuerdos con EEUU y con la Unión Europea, sino que el instrumento apuesta a ser el medio para una profundización económica y comercial con Asia Pacífico (Bartesaghi, 2013).

Otra de las características tiene que ver con su estructura institucional, donde a diferencia de los procesos de integración clásicos de la región, se determinó que el bloque posea un nivel de burocracia mínimo, apostando al pragmatismo y flexibilidad a la hora de la toma de decisiones. Esta realidad, sumado al importante número de reuniones y la velocidad mostrada en la toma de decisiones por parte de los países miembros, posicionó al acuerdo como exitoso en términos comparados, si bien los logros son aún poco significativos.

Como puede observarse, América Latina y el Caribe ha ido construyendo con el correr de las últimas décadas, una densa red de procesos de integración y de discusión política (muchos de los cuales no fueron considerados en este documento por tratarse de organismos que no tuvieron en su origen un objetivo económico y comercial) que incluso o se han superpuesto, o han generado ciertas contraposiciones entre los modelos. Este último fenómeno se da especialmente desde la creación del ALBA en adelante y con la conformación de la Alianza del Pacífico, dos procesos que impusieron una nueva lógica integracionista en la región, que llevó a ciertas tensiones que se siguen procesando hasta el presente.

\section{La institucionalidad y la cohesión como factores de éxito en los procesos de integración. Tres casos de estudio: MERCOSUR, ALBA y Alianza del Pacífico}

La definición del universo de procesos de integración considerados para este capítulo, contempla parte los diferentes modelos que han ido emergiendo en los últimos años en la región.

Uno de los procesos de integración, más particularmente el caso del MERCOSUR, representa la modalidad clásica de integración latinoamericana que siguió, si bien mucho tiempo después, el mismo modelo de la Unión Europea, confeccionando como fue anteriormente comentado un mercado común. Este acuerdo podría también ubicarse en la misma categoría del MCCA y del CARICOM, si bien los tres han registrado diferentes niveles de éxito en su desarrollo.

Los otros dos procesos de integración incluidos en el análisis, representan un tipo de integración que algunos expertos han identificado como una posible nueva fase, aunque debe tenerse en cuenta que se trata de procesos bien distintos entre sí, tanto por sus perfiles políticos como por sus instrumentos comerciales. Además, tanto el ALBA como la Alianza del Pacífico se han presentado como dos iniciativas contrapuestas, especialmente en lo que tiene que ver con el modelo. Los primeros y liderados por Venezuela, como representantes del denominado Socialismo del Siglo XXI, mientras que a los miembros de la Alianza del Pacífico se los identifica como economías liberales muy afines a los intereses de Washington.

Las variables relevadas analizan el número de miembros, el nivel de profundidad institucional, la aspiración en el nivel de integración y las áreas y proyectos de cooperación.

Respecto a los miembros, se observan algunas características que adelantan las particularidades de los procesos de integración en cuanto a sus orígenes así como su funcionamiento. En el caso del MERCOSUR, inicialmente tuvo cuatro miembros, si bien luego se incorporó Venezuela, mientras que Bolivia se encuentra en proceso de integrarse plenamente al bloque.

Naturalmente es Brasil la potencia de mayor importancia relativa del bloque en términos de su población, territorio o PIB, lo que marcó el bloque desde los orígenes, especialmente en su estratégica relación con Argentina, socio principal de Brasil en Sudamérica. Debe tenerse en cuenta que el país norteño le exportó más de US\$ 14.000 millones a su vecino en 2014, monto que se equipara con lo exportado por la potencia regional a Venezuela, México, Paraguay y Uruguay computados en su conjunto. Si bien la inclusión de nuevos miembros podría equilibrar las asimetrías existentes en el bloque, dadas las características de los nuevos integrantes y en especial la modalidad en que se han incorporado (en el caso de Venezuela incumpliendo los procedimientos jurídicos vigentes, mientras que en el caso de Bolivia por la incertidumbre que genera la doble membrecía, dado que dicho país integra también la Comunidad Andina, la que ya ha aclarado no abandonará), dicho efecto parece poco probable.

En el caso de la Alianza del Pacífico, también existe una importante asimetría dado la presencia de México, mientras que los otros tres miembros muestran una importancia relativa al menos un poco más homogénea (especialmente entre Chile y Colombia). Ahora bien, la diferencia con el MERCOSUR tiene que ver con que México no lideró el proceso de integración ni posee una relación estratégica con ninguno de los miembros de la Alianza del Pacífico. En efecto, su incorporación se encuentra relacionada con una estrategia de inserción internacional que lo posiciona como posible puente entre Asia Pacífico, Estados Unidos y América Latina por su avanzada estructura productiva, lo que lo ubica adecuadamente en las cadenas globales de valor. La importancia de México en la Alianza tiene que ver también con aspectos geopolíticos, ya que cualquier acción directa o indirecta de este país en Sudamérica es observada con cierto recelo por parte de Brasil.

Por último en el caso del ALBA, la participación de Venezuela es excluyente. Este proceso que tiene un objetivo político, pero potenciado por la diplomacia basada en el petróleo, surge principalmente de una estrecha relación política y también económica entre Venezuela - y en particular de Chávez con $\mathrm{Cuba}^{5}$, para mitigar los impactos del bloqueo de Estados Unidos a la isla.

5 Ver acuerdo bilateral firmado entre Venezuela y Cuba en el año 2004 en la ciudad de la Habana, denominado Acuerdo para la Aplicación del ALBA. 
El ALBA es un proceso de integración que no ha conformado instituciones más allá de las que dependen de Venezuela, por lo que las asimetrías en este bloque son de suma importancia en todas las variables. Quizás la única excepción es la política, donde Cuba adquiere a nivel internacional un papel de preponderancia histórica debido a su relación con Estados Unidos, lo que lo posiciona en el ALBA como un miembro de importancia relativa solo detrás de Venezuela, que es la locomotora del proceso a partir de sus recursos petroleros. El resto de los miembros con la excepción de Ecuador, país con un mayor desarrollo relativo, son pequeñas economías dependientes de la ayuda internacional y con problemas económicos y sociales estructurales, lo que las hace muy dependientes de los programa de apoyo internacional como los ideados por Venezuela en el marco del ALBA. La cercanía de estos territorios a la zona de influencia de Estados Unidos es parte del interés geopolítico de Venezuela en el proceso, el que opera a través de acciones bilaterales con cada uno de los miembros.

En cuanto a la profundidad institucional, el relevamiento comparativo indica que el MERCOSUR es indiscutidamente el bloque con un mayor nivel de profundidad institucional y con la mayor aspiración en términos de sus instrumentos de integración, ya que aspira a conformar un mercado común, con la previa conformación de una zona de libre comercio y una unión aduanera.

Este punto es resaltado ya que algunos autores entienden que la integración denominada profunda favorece la realización de las reformas internas exigidas por el proceso y lo que es más importante, su posterior aceptación (Venables, 2000; Polonia, 2003). El MERCOSUR a su vez, posee una densidad institucional que supera ampliamente a la del ALBA y la de la Alianza del Pacífico, ya que posee órganos con capacidad decisoria, los que incluso aprueban normas de forma directa - sistema supranacional -, si bien debe reconocerse que dichas normas refieren solo a aspectos formales y no a temas de importancia económica y comercial, una secretaría permanente con funciones específicas, un sistema de solución de controversias con una instancia permanente en los casos de revisión (TPR), un Parlamento, el que está en proceso de consolidación y también órganos derivados, caso del Fondo de Convergencia Estructural del MERCOSUR (FOCEM), sistema que suele ser destacado como uno de los de mayor avance en los años recientes (Bartesaghi, 2015).

\section{Principales características de los procesos de integración considerados}

\begin{tabular}{|c|c|c|c|c|}
\hline \multicolumn{2}{|c|}{ Cantidad de miembros } & 5 & 11 & 4 \\
\hline \multirow{3}{*}{$\begin{array}{l}\text { 'rofundidad } \\
\text { nstitucional }\end{array}$} & $\begin{array}{l}\text { Sistema de Solución de } \\
\text { Controversias }\end{array}$ & $\begin{array}{c}\text { Regulado por el Protocolo de Olivos. Cuenta } \\
\text { con un Tribunal Permanente de Revisión y } \\
\text { Tribunales Arbitrales Ad Hoc. }\end{array}$ & No cuenta & No cuenta \\
\hline & Secretaría & $\begin{array}{l}\text { Se crea con el Tratado de Asunción y su } \\
\text { principales funciones eran la guarda de } \\
\text { documentos y dependía del Grupo Mercado } \\
\text { Común. En el protocolo de Ouro Preto se la } \\
\text { incluye en la estructura institucional. }\end{array}$ & $\begin{array}{c}\text { Coordinación permanente del ALBA } \\
\text { (secretaria ejecutiva) }\end{array}$ & No cuenta \\
\hline & Parlamento & $\begin{array}{c}\text { Creado en el } 2006 \text { Protocolo Constitutivo del } \\
\text { Parlamento del MERCOSUR }\end{array}$ & No cuenta & No cuenta \\
\hline \multicolumn{2}{|c|}{ Aspiración en el nivel de integración } & Mercado Común. & No especifica. & No especifica. \\
\hline \multicolumn{2}{|c|}{ Áreas y proyectos de cooperación } & $\begin{array}{l}\text { Dos modalidades de cooperación: Intra- } \\
\text { MERCOSUR y Extra-MERCOSUR. Las áreas } \\
\text { prioritarias son: • Políticas sociales regionales, } \\
\text { en el ámbito del Plan Estratégico de Acción } \\
\text { Social del MERCOSUR (PEAS). } \\
\text {-Participación de las organizaciones sociales } \\
\text { en el proceso de integración. } \\
\text { •Cooperación en materia cultural, audiovisual } \\
\text { y de apoyo a la sociedad de la información. } \\
\text { •Ciencia, innovación tecnológicay } \\
\text { capacitación. } \\
\text { •Integración productiva. } \\
\text { •Inocuidad de los alimentos. } \\
\text { •Transporte. } \\
\text { •Estadística. } \\
\text { •Medio Ambiente. }\end{array}$ & $\begin{array}{c}\text { Proyectos Grannacionales: Programa de } \\
\text { acción dirigido a cumplir con los } \\
\text { principios y fines del ALBA, validado } \\
\text { por los países integrantes y cuya } \\
\text { ejecución involucre a dos o más paises, } \\
\text { para beneficio de las grandes mayorías } \\
\text { sociales. Existen también las empresas } \\
\text { de los paises ALBA integradas } \\
\text { productivamente, cuyas producciones } \\
\text { se destinarán fundamentalmente al } \\
\text { mercado INTRA-ALBA, para configurar } \\
\text { una zona de comercio justo y cuya } \\
\text { operación se realizará de forma } \\
\text { eficiente. Las áreas tratadas } \\
\text { actualmente en estos proyectos son: } \\
\text { alimentación, ambiente, ciencia y } \\
\text { tecnología, comercio justo, cultura, } \\
\text { educación, energía, industria y minería, } \\
\text { salud, telecomunicaciones, transporte y } \\
\text { turismo. }\end{array}$ & $\begin{array}{c}\text { - Plataforma de movilidad estudiantil y } \\
\text { academica. } \\
\text { - Proyecto diplomacia deportiva. } \\
\text { - Programa de voluntario juvenil de la } \\
\text { Alianza del Pacífico. } \\
\text { - Proyecto "La Cocina Tradicional } \\
\text { Sostenible como Elemento de Intercambio } \\
\text { Cultural y Proyección de los Países } \\
\text { Miembros de la Alianza del Pacífico". } \\
\text { - Proyecto "Integración de Fomento a la } \\
\text { Producción y Consumo Sustentable - } \\
\text { PyCS". } \\
\text { - Cooperación Cientíica en Materia de } \\
\text { Cambio Climático en la Alianza del } \\
\text { Pacífico: Monitoreo de la Biodiversidad. }\end{array}$ \\
\hline
\end{tabular}

Fuente: elaboración propia en base a los sitio web oficiales del Mercosur, ALBA y Alianza del Pacífico. 
En el caso de los otros dos procesos de integración considerados, el nivel de institucionalidad es especialmente bajo, lo que contradice de cierta forma la ambiciosa meta de alcanzar una "integración profunda" como lo plantea por ejemplo el acuerdo fundador en el caso de la Alianza del Pacífico. Es más, este último proceso de integración hizo especial hincapié en que el bloque no fomentaría la creación de una institucionalidad profunda como la existente en otros procesos característicos de la región. Algunos autores entienden que se trata de una contradicción, ya que parece dificultoso alcanzar las metas propuestas por los miembros de la Alianza sin constituir instituciones supranacionales (Tremolada, 2013).

Ahora bien, más allá de que indiscutidamente el MERCOSUR es el proceso con un mayor nivel de institucionalidad, lo que favorece de acuerdo a la teoría a la mayor cohesión entre los miembros y el mayor desarrollo del proceso, la ineficiencia de algunos de los órganos anteriormente presentados, puede afectar dicho objetivo. Este es el caso del incumplimiento de algunas de las normas aprobadas por los órganos con capacidad decisoria del bloque o el no cumplimiento de algunos de los laudos arbitrales, lo que afecta profundamente la credibilidad de las instituciones.

Los autores que señalan la importancia de la institucionalidad en los modelos de desarrollo de los países o en los procesos de integración, parten del supuesto de que las instituciones creadas cumplen con sus objetivos principales de forma eficiente, lo que acarrea como resultado un respeto por parte de la comunidad involucrada. Este aspecto ha sido una de las virtudes destacadas por la Unión Europea, especialmente en momentos en que la crisis económica no logró arrastrar a las instituciones europeas, las que le han puesto un coto a las corrientes de opinión que visualizaban una crisis de esa naturaleza. Las instituciones europeas lograron alcanzar el respeto por parte de los ciudadanos, lo que favoreció un nivel de cohesión que naturalmente se encuentra ausente en la región, que como es sabido cuenta con contados antecedentes de desestabilización institucional por crisis económicas.

Los programas de cooperación son un vehículo para aumentar la interrelación entre los Estados miembros. En este campo, los países socios cuentan con la posibilidad de aplicar ciertas innovaciones con flexibilidades que pueden lograr una conexión entre las partes que muchas veces supera la alcanzada por la institucionalidad más tradicional. El efecto en este tipo de programas suele ser mayor en los procesos de integración donde existe un mayor nivel de asimetrías entre los socios o en los casos en que se destaca la presencia de un líder indiscutido, tanto en la conformación de la iniciativa como en el desarrollo.

Los tres procesos analizados poseen programas de cooperación, si bien en el caso del ALBA, se trata de definiciones más generales, relacionadas en mayor medida con políticas de desarrollo, más que con mecanismos específicos. Este aspecto no debería llamar la atención si se tiene en cuenta la ya comentada asimetría que caracteriza al proceso de integración.
Los temas abordados por el MERCOSUR en las denominadas por este estudio como áreas y proyectos de cooperación, así como ocurre en el análisis del nivel institucional, parecen ser más profundos en términos temáticos, lo que evidencia la maduración del proceso respecto al tipo de programa presentado por la Alianza del Pacífico, atendiendo a temas que podrían indicar un menor nivel de profundidad en cuanto a las temáticas abordadas, lo que así como se mencionó en el caso de la institucionalidad asociada al bloque, podrían ser considerado una contradicción si se tiene en cuenta que los miembros pretenden alcanzar una integración profunda, lo que de acuerdo a los objetivos definidos en el tratado originario implicaría conformar un mercado común.

\section{Conclusiones}

América Latina cuenta con un número importante de iniciativas integracionistas, las que presentan como pudo observarse diferentes características. Un análisis del alcance de cada proceso y en especial los tres casos de estudio presentados, permiten identificar las diferencias en cuanto al alcance institucional o los programas de cooperación.

Más allá de las particularidades de cada proceso, puede concluirse que el nivel institucional de los procesos de integración no necesariamente garantizan el éxito en la consecución de los objetivos, ya que si bien el MERCOSUR posee un nivel institucional y de programas de cooperación más profundo que los otros dos procesos comparados, caso del ALBA y la Alianza del Pacífico, por diferentes razones estos dos procesos poseen un nivel de cohesión mayor, especialmente por una visión ideológica común, caso del ALBA o por mayores afinidades en las políticas económicas en el caso de la Alianza del Pacífico.

Si bien la institucionalidad es clave para alcanzar una integración de corte más profunda, la que pretende ser alcanzada en el MERCOSUR, pero no necesariamente en los otros dos modelos, para cumplir con dicho objetivo será necesario contar con un liderazgo, el que debería recaer en Brasil, que no lo ha ejercido especialmente en los momentos clave. También es necesario innovar en los mecanismos para alcanzar las metas, lo que ha estado ausente en el MERCOSUR, que no ha logrado constituir una institucionalidad eficiente o con las flexibilidades necesarias para impedir que se consolide la sensación de fracaso, y como resultado, que no se respete la institucionalidad del bloque como medio apropiado para el desarrollo de los miembros, lo que termina como resultado afectando el nivel de cohesión regional.

Por otra parte, si bien tanto la Alianza del Pacífico como el ALBA lograron un nivel de afinidad política o económica mayor que el presentado entre los miembros del MERCOSUR, la falta de institucionalidad y la ejecución de programas profundos de cooperación, podrían afectar el desarrollo del proceso y especialmente la profundidad de las interacciones estatales, si pierde fuerza la voluntad política que hoy mantiene la firmeza de las iniciativas. En el caso del ALBA, los cambios políticos a partir de la ausencia del liderazgo ejercido por Chávez, los problemas internos de Venezuela, sumado a la profundización de la crisis económica a partir de la caída en el precio internacional del petróleo, podrían afectar la evolución de la iniciativa. 
Asimismo, la Alianza del Pacífico deberá confirmar la sensación de éxito que generó la iniciativa, especialmente por el impacto internacional y regional a partir del interés del importante número de países observadores. Dicho bloque que pretende alcanzar un nivel profundo de integración, pero con un enfoque pragmático y con la ponderación de los intereses económicos más que políticos, lleva al proceso a enfrentarse al desafío de cumplir con dichas expectativas con un reducido nivel de institucionalidad y aprobación de programas de cooperación.

Si bien un ejercicio prospectivo respecto a los tres procesos es dificultoso, dada la experiencia comparada, existen algunos pilares que siguen estando muy presentes en todos los esquemas a nivel internacional, como el caso de la importancia de una institucionalidad, que por cierto debe ser eficiente y cumplir con sus atribuciones, la innovación de los actores para solucionar los inevitables conflictos que se presentarán durante la integración de los socios y el necesario liderazgo de los miembros de mayor importancia relativa en los bloques.

Todos estos aspectos singuen siendo centrales para el desarrollo sustentable de los países a nivel mundial, especialmente en momentos en que las dinámicas actuales llevan a tensiones con impactos que aún pueden ser desconocidos. Es esperable que las diferentes iniciativas regionales profundicen los vínculos a los efectos de alcanzar un desarrollo sustentable en América Latina y el Caribe.

\section{Agradecimientos}

A la Universidad Católica del Uruguay, y en especial a la Facultad de Ciencias Empresariales por el apoyo brindado en las tareas de investigación emprendidas por el Departamento de Negocios Internacionales e Integración.

A los asistentes del 21 Congreso Anual de IAJBS por los valiosos aportes que enriquecieron esta investigación.

\section{Bibliografía}

Bartesaghi, I. (2011). El Mercado Común del Sur 1991 -2010. Montevideo, Uruguay: Cámara de Industrias del Uruguay.

Bartesaghi, I. (2012). La evolución de la institucionalidad europea: El caso del Parlamento europeo. Revista Relaciones Internacionales. Universidad de la Plata. Vol. 21, núm. 42, pp. 39 -63.

Diez de Velasco, M. (2002). Las organizaciones internacionales. (12a ed.). Madrid, España: Tecnos.

Lopez, G. (2012). El Mercado Común Centroamericano. Mediterráneo Económico 22, pp 261 -269.

Quitral Rojas, M. (2009). La integración económica latinoamericana en tiempos de crisis: alcances y limitaciones para su consolidación. Nueva Sociedad. No. 22, pp. 30 - 40.
Romero, A. (2013). CARICOM: Evolución económica, relaciones con Europa e integración regional. Aportes, Revista de la Facultad de Economía, BUAP. Año XVIII, Número 49.

Sanahuja, J. (2007). Regionalismo e integración en América Latina: perspectivas y balances. Pensamiento Iberoamericano. No. 0, segunda época, pp.75 -106.

Tremolada, E. (2013). La Alianza del Pacífico: ¿una organización consecuente con tus objetivos? En Repensando la integración y las integraciones (Tremolada, E. editor). Universidad Externado de Colombia, Bogotá, Colombia.

Venables, A. (2000). International trade; regional economic integration. Preparado para la International encyclopedia of social and behavioral sciences, London School of Economics.

\section{Recursos electrónicos}

Bartesaghi, I. (2013). La encrucijada de la política exterior del Uruguay: ¿el Mercosur, la Alianza del Pacífico o ambas? Presentado en XXVII Congreso AMEI, octubre 2013. http://www.ucu.edu.uy/sites/default/files/La\%20encrucijada\%20de\%20la\%20pol\%C3\%ADtica\%20 exterior\%20del\%20Uruguay.pdf

Bartesaghi, I. (2015). El MERCOSUR, 24 años después. Informes sobre integración económica $\mathrm{N}^{\circ} 1$. Departamento de Negocios Internacionales e Integración. http://www.ucu.edu.uy/sites/default/files/ pdf/2015/ISIE_Mercosur_N\%C2\%B01.pdf

Polónia, S. (2003). Mercosur: dilemas y alternativas de la agenda comercial. Documento de trabajo -IECI- 06c. INTAL. http://idbdocs. iadb.org/wsdocs/getdocument.aspx?docnum $=33036462$

Polónia, S. (2003). Mercosur: en busca de una nueva agenda. INTAL ITD -BID. http://publications.iadb.org/bitstream/handle/11319/2617/ MERCOSUR\%3A\%20en\%20busca\%20de\%20una\%20nueva\%20agenda.\%20MERCOSUR\%3A\%20dilemas\%20y\%20alternativas\%20de \%20 la\%20agenda\%20 comercial.pdf?sequence $=1$

SELA (2014). Evolución de la Alianza Bolivariana para los Pueblos de Nuestra América -Tratado de Comercio de los Pueblos (ALBA-TCP). Secretaría Permanente del SELA. Caracas, Venezuela. http://www. sela.org/attach/258/EDOCS/SRed/2014/09/T023600006055-0-Di_ No._4_-_ALBA-TCP_doc_Formato_3.pdf

\section{Páginas web consultadas}

Alianza Bolivariana para los Pueblos de Nuestra América: http://alba-tcp.org/

Mercado Común del Sur: http://www.mercosur.int/

Alianza del Pacífico: http://alianzapacifico.net/ 